\title{
The liquid barrier filter: total protection against particulate contaminants
}

\author{
WD SEUFERT, FRANCE BESSETTE, AND G LACHIVER \\ From the Département de biophysique, Faculté de médecine, Université de Sherbrooke, Sherbrooke, Québec, \\ Canada J1H $5 \mathrm{~N} 4$
}

\begin{abstract}
The design, construction, and operation of a new type of filter that eliminates particulate contaminants from a current of gas is described. It has a yield higher than that of any conventional filter or filtration system. It is believed that a liquid barrier filter provides the best possible protection in all cases where not even a small number of toxic particulates can be tolerated in an atmosphere.
\end{abstract}

The permeability of some perfluorocarbon liquids to gases is so high that one can literally breathe through them. Small mammals submerged in a perfluorocarbon that is in contact with a normobaric atmosphere exchange sufficient oxygen and carbon dioxide to satisfy their metabolic requirements ${ }^{1}$ despite the fact that the animals' lungs are filled with the liquid and although the diffusion pathway is very long, extending from the air/liquid interface to the alveolar surface. The continuity of the liquid phase is not impaired in these experiments-that is, the air passes through it by diffusion only. There is no convective gas flow, and neither permanent nor transient discontinuities (neither pores nor bubbles) exist in the liquid.

A contiguous liquid phase with a gas permeability high enough to sustain respiration should constitute the ideal filter against particles contaminating an atmosphere. Gases will dissolve in the liquid and pass through it by diffusion, but particulate contaminants must collide with it, lose their momentum in the impact, and be caught. We have shown that filters of this type approach the ideal collection yield. ${ }^{2}$ Conventional filters always provide an uninterrupted though tortuous pathway for the gas, and their performance characteristics are, therefore, determined by the probabilities of capturing a contaminant and holding it on the filter in the face of the gas stream. ${ }^{3}$ A particle's dynamic flow characteristics relative to the obstructions put into its path are important in all conventional filtration mechanisms (matrix or fabric filters, electrostatic filters, or scrubbers). By contrast, a liquid barrier filter cap-

Received 9 May 1983

Accepted 13 June 1983 tures particulates regardless of their ingress velocity, their shape, size, or nature, and eliminates them from a current of gas by reducing their kinetic energy through collisions with the liquid's constituent molecules.

The liquid phase is an insurmountable barrier to all particulates that cannot muster sufficient kinetic energy to tunnel through it. Therefore, a liquid barrier filter's minimum thickness is determined by both (and only) the mass and the velocity of the incoming particles. We see that small particles need not concern us at all: their small momentum on collision is immediately transferred to the liquid, and they are stopped. In conventional filter systems particles below a certain size are difficult to eliminate from a flowing gas, and these filters have an upper and a lower size limit to their operational efficacy. ${ }^{4}$

The maximum thickness of a liquid barrier filter is determined by the perfluorocarbon's permeability to a given gas-that is, by the product of its solubility and its diffusion coefficient in the liquid. If the filter is to be used to protect people from particles in the respired air an inspiratory pressure of about $5 \mathrm{~cm}$ $\mathrm{H}_{2} \mathrm{O}$ will have to permit the transfer, by diffusion, of approximately 100 litres of air a minute.

\section{Particle penetration of a liquid phase}

As we have shown earlier, ${ }^{2}$ a thin phase of a liquid perfluorocarbon absorbed into an inert glass fibre matrix stops particles with nearly ideal efficacy even when they bombard the filter at high speeds. To our knowledge, the trajectories of particles entering a liquid of a given density have not yet been examined systematically as a function of particle mass, size, shape, and ingress velocity. We can, however, calcu- 
late the maximum depth to which a solid particle should penetrate into a liquid under ideal conditions.

Collisions of solids impinging on a liquid surface are inelastic since some of the kinetic energy is lost as heat through the generation of waves or by deformations of the colliding partners. The influence of these parameters separately on the depth of penetration cannot yet be assessed because of the lack of published data, but we can calculate the maximum possible penetration distance in the following way. At the instant of an elastic collision, the momentum of a particle of mass $\mathrm{m}_{p}$ and velocity $\mathrm{v}_{p}$ is equal to the momentum of the liquid of mass $m_{l}$ that is displaced at velocity $\mathrm{v}_{l}$ :

substituting,

$$
\mathrm{m}_{p} \mathrm{v}_{p}=\mathrm{m}_{l} \mathrm{v}_{l}
$$

$$
\mathrm{d}_{p} \mathrm{~V}_{p} \mathrm{v}_{p}=\mathrm{d}_{l} \mathrm{~V}_{l} \mathrm{v}_{l}
$$

where $d$ is the density and $V$ the volume. In an ideally elastic collision $\mathrm{v}_{p}=\mathrm{v}_{l}$-that is, the velocity imparted on the resting liquid during the collision-equals that of the incoming projectile. The diameter of the particle and that of the discontinuity introduced in the liquid on penetration are also equal; we thus replace $V_{p}$ by $L_{p}$, the effective fluid dynamic length ( $=$ the dimension of length in the direction of the driving current) of the penetrating particle, and $V_{l}$ by $L_{l}$, the depth of penetration, to obtain

$$
\mathrm{L}_{\mathrm{l}}=\frac{\mathrm{d}_{p}}{\mathrm{~d}_{l}} \mathrm{~L}_{p} \text {. }
$$

Since the collision is inelastic, the liquid yields to the impact with a speed much lower than the particle's entry velocity, and the kinetic energy is spent more rapidly than is predicted by the considerations above. Consequently, incoming particles are stopped in reality within distances well below their fluid dynamic length if their density is equal to, or below, that of the substrate penetrated. Perfluorocarbon liquids have densities between 1.6 and $1.9 \mathrm{~g} / \mathrm{cm}^{3},{ }^{5}$ and they should therefore arrest, for example, even solid plutonium contaminants (density $16 \mathrm{~g} / \mathrm{cm}^{3}$ ) within about ten times the particle length. A very thin perfluorocarbon phase assuredly eliminates all particulates from the inspired air.

The series of high speed photographs (fig 1) shows that perfectly spherical polysaccharide particles (buoyant density about $1.1 \mathrm{~g} / \mathrm{cm}^{3}$ ) cannot enter into perfluorotributylamine (density $1.87 \mathrm{~g} / \mathrm{cm}^{3}$ ) but remain at its surface even when shot into the liquid at a velocity of $312 \mathrm{~cm} / \mathrm{s}$. The particle whose impact is shown does not submerge into the liquid but remains visible at the surface all through the filmed sequence.

\section{Gas permeation through the perfluorocarbon phase}

The transfer of air through the liquid phase constituting the barrier for particulates can proceed at a rate fast enough to replenish the oxygen consumed metabolically only if oxygen has a high partial solubility in the liquid to be used as a filter. In the absence of convection, air will have to dissolve first in the liquid in proportion to the ambient pressure; it will then diffuse across the liquid phase to be extracted on the other side, according to the prevailing pressure gradient. The determinants for an adequate transfer are, therefore, the solubility and the diffusion coefficient of air in the filtering liquid. We attempt to estimate, firstly, under which conditions

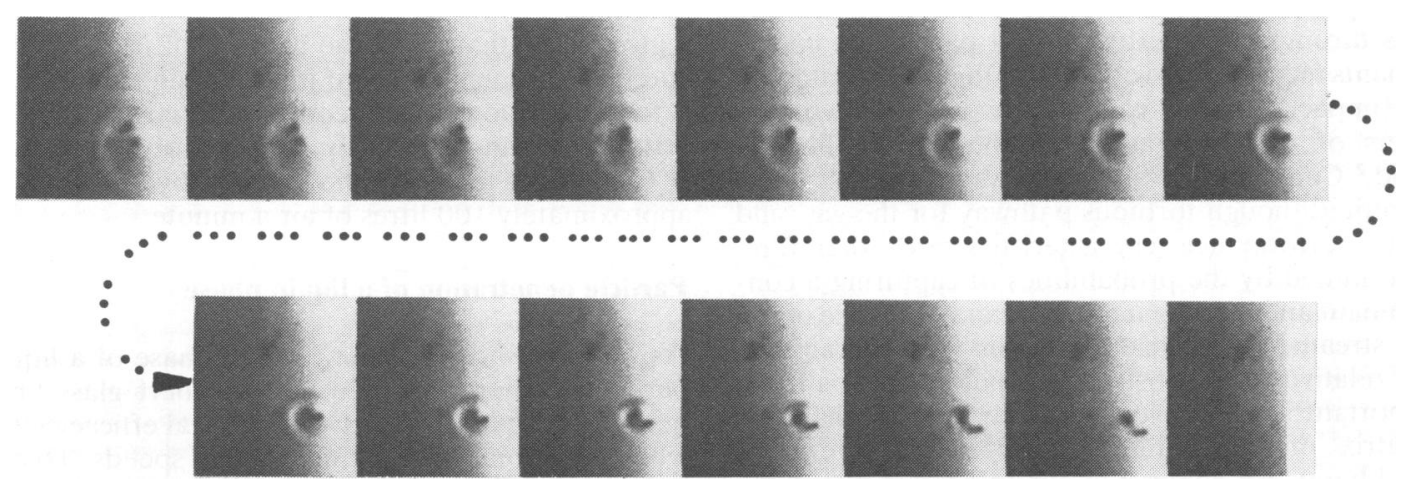

Fig 1 High speed impact of spherical polysaccharide particles with surface of perfuorocarbon liquid. Diameter of particles between 1 and $4 \times 10^{-5} \mathrm{~m}$. Ingress velocity $312 \mathrm{~cm} / \mathrm{s}$. Hycam camera; $10000 \mathrm{frames} / \mathrm{s}$. 
one could breathe through a thin phase of a perfluorocarbon liquid completely saturated with air. An equation based on Fick's First Law of diffusion expresses gas transfer in terms of solubility and diffusivity in the liquid:

$$
\frac{\mathrm{dn}}{\mathrm{dt}}=-\mathrm{A} \mathrm{D} \frac{\alpha \Delta \mathrm{p}}{22 \cdot 4 \Delta \mathrm{x}}
$$

where $\mathrm{dn} / \mathrm{dt}$ gives the number of moles of gas diffusing across the liquid phase per second at STP; $A$ is the area of exchange, $\mathrm{D}$ the diffusion coefficient, $\alpha$ the Bunsen solubility coefficient, $\Delta \mathrm{p} / \Delta \mathrm{x}$ the partial pressure gradient (in atm), linear throughout the liquid if the gas does not react with it. The gradient is the driving force for transfer.

We have found that the diffusivities of respiratory gases in perfluorotributylamine are, with values between 1.3 and $2.0 \times 10^{-5} \mathrm{~cm}^{2} / \mathrm{sec}$, of the same order of magnitude as in water. ${ }^{6}$ Nevertheless, the solubilities of gases are greater in fluorocarbons than in any other liquid. In fact, some perfluorocarbon liquids can dissolve up to $50 \mathrm{ml}$ of oxygen per $100 \mathrm{ml}$ at room temperature and under $1 \mathrm{~atm}$ of $\mathrm{O}_{2}{ }^{7}$ Their oxygen carrying capacity is thus more than twice as high as that of normal whole blood. Data on the solubility of standard air in perfluorocarbons are not available, but the partial solubility of oxygen taken up from air at room temperature (expressed as the Bunsen coefficient) is about $0 \cdot 1$, and that of nitrogen taken up from air is about $0 \cdot 25 .^{7}$ We therefore use a value of 0.3 as the typical solubility of air in a perfluorocarbon liquid. We also note that the partial oxygen solubility is considerably higher than would correspond to its partial pressure in a normal atmosphere.

The volume of required air should be delivered through the filter with an inspiratory effort not greter than that necessary to raise $5 \mathrm{~cm}$ of water. The thickness of the liquid phase can be held consistently at $10^{-3} \mathrm{~cm}$. Perfluorocarbon liquids are highly surface active; they adsorb and cling readily to a fine mesh stainless steel support. Their surface activity is a very desirable quality in our application, and we have observed that these liquid films can withstand, on a fine support mesh, sustained gas pressure differences of $50 \mathrm{~cm} \mathrm{H}_{2} \mathrm{O}$.

Exploring the possibility of incorporating a liquid barrier filter in a portable respirator, we estimate that a total exchange surface of $7500 \mathrm{~cm}^{2}$ can be enclosed easily in a canister not more than $15 \mathrm{~cm}$ in diameter and $20 \mathrm{~cm}$ high. We allow for some functionally less efficient areas and use $7 \times 10^{3} \mathrm{~cm}^{2}$ as the total exchange area in Fick's equation. Inserting these values, and a diffusion coefficient for air of approximately $1.5 \times 10^{-5} \mathrm{~cm}^{2} / \mathrm{sec}$, we obtain slightly less than 10 litres of air transferred per minute, a value that is too small by a factor of 10 . In other words the inspiratory pressure would have to be raised to physiologically intolerable levels in order to draw a sufficient volume of air through the filter. We can, however, decrease the inspiratory load by a fan such as is used, for example, in the portable power filtration unit recently described by Clark and Schwabe. ${ }^{8}$ The use of a fan has the additional advantage that the physiological pressure variations during the respiratory cycle are evened out. With a pressure difference of $50 \mathrm{~cm} \mathrm{H}_{2} \mathrm{O}$, the design objective of an air flow of 100 litres/min across the liquid barrier filter would be attained. This is only about $1 / 20$ of an atmosphere (or $0.8 \mathrm{psi}$ ) and is easily furnished by a portable power unit. The higher differential solubility of oxygen compared with other gases taken up from air has been neglected in these considerations but would, of course, favour the conclusion that a portable respirator incorporating a liquid barrier filter is entirely feasible. The resistance of a powered device (= inspiratory driving force/gas flow) falls within acceptable limits.

We see as the most important advantages of liquid barrier filters the fact that they can provide highly effective personal protection against a wide variety of highly toxic particulate contaminants in an atmosphere. The design considerations for stationary liquid barrier filters are different from those given here since more options are available as to the means to maintain a required flow of gas.

\section{Design of a portable respirator containing a liquid barrier filter}

Figure 2 shows one possible design for a portable respirator. The canister containing the liquid barrier filter is connected to the face mask through a standard inhalation valve. The filtering unit consists of 54 lamellae $\left(140 \mathrm{~cm}^{2}\right.$ each) that are formed from a fine mesh stainless steel fabric $\left(10^{-3} \mathrm{~cm}\right.$ thick; 36 square cells per $\mathrm{mm}^{2}$ ) arranged in 27 radial pleats over a wire frame. This filtering unit is held between an inner and outer perforated metal cylinder. At the top of the canister is a reservoir containing 200 to $250 \mathrm{ml}$ of the perfluorocarbon filtering liquid. The bottom of the reservoir is made of a perforated metal plate that is covered on its outside by the same fine mesh material also used for the pleats. A brush seal at the top of each pleat contacts the bottom of the fluid reservoir. The wire mesh is so dense that the surface active liquid can drain through it only where it is touched by the brush. The same brush is also attached to the bottom of the pleats to seal them against the filter casing. The perfluorocarbon liquid seeps on to the pleats and forms an uninterrupted film that is continually renewed, when the 


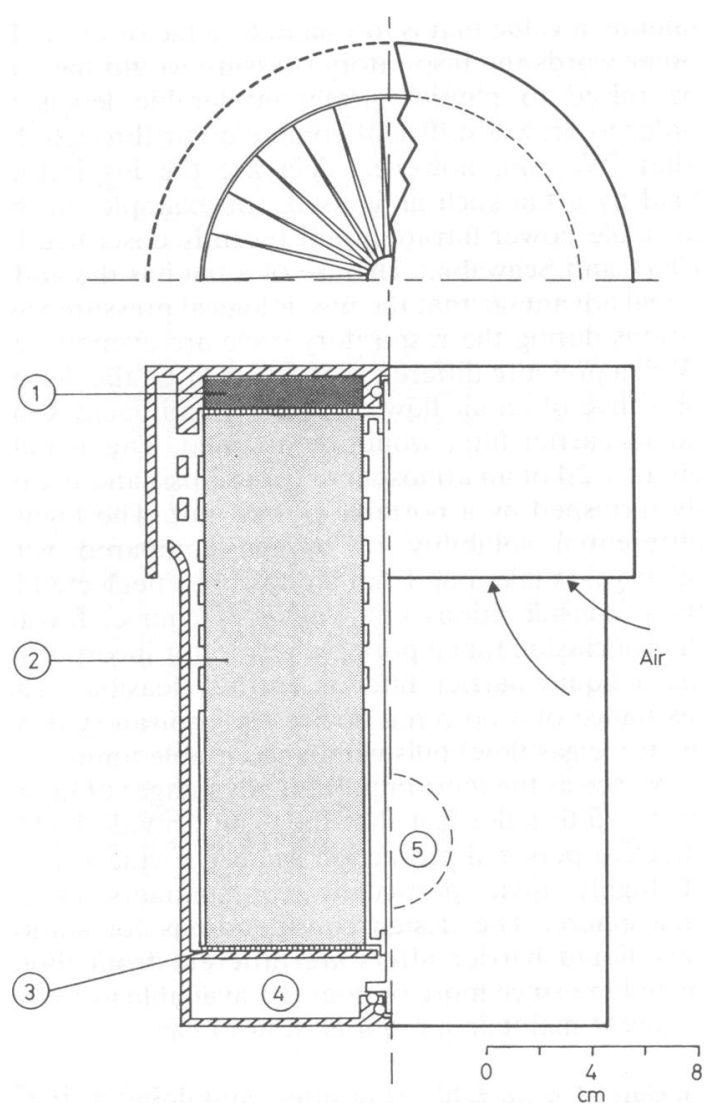

Fig 2 Design of a portable canister incorporating a liquid barrier filter (1) reservoir, filtering liquid; (2) liquid barrier filter support pleats; (3) brush seal; (4) space for driving mechanism or reduction gear; and (5) gas outlet.

filtering unit is slowly rotated, by drainage from the reservoir. The continuous slow vertical flow of liquid on the pleats ensures that a clean filtering surface is always presented to the incoming air as the particles deposited on the filter are washed off. The liquid barrier filter thus cannot be overloaded within its planned operational lifetime, a very important advantage over conventional filters. The pleats of the support matrix touch the covers that contain them; this ensures that excess perfluorocarbon liquid is properly drained from the spaces where the angle between pleats becomes very small. The air flows through the pleats and is collected in the central channel of the filtering unit as shown. The fan rotor (not illustrated), which reduces the inspiratory resistance as discussed, is placed in the hose between the filter canister and the face mask. The filter unit is rotated by the fan motor through a reduction gear, or by a separate drive.

Some of the filtering liquid will evaporate into the inspired air. It is to be noted, however, that perfluorocarbons, in general, are chemically inert. Perfluorotributylamine in particular, the substance with which we have done our experiments, is completely non-toxic; it is, in fact, used in the formulation of "artificial blood." A volume of about $200 \mathrm{ml}$ of filtering liquid is sufficient for the geometry proposed here, and the liquid barrier filter would perform at its full efficacy of particulate removal during approximately one hour, after which period the contaminated canister should be replaced. The filtering liquid can be purified for repeated use.

A liquid barrier filter can be attached to a face mask in series with other cartridges containing conventional filters formulated against, for example, toxic gases should the need arise. It can also be used in conjunction with a "roughing" filter as a first stage which would already eliminate some of the contaminants. This latter arrangement has the advantage that the first stage would then also serve as a baffle or shield for the second and protect the liquid barrier filter against disruption by explosive pressure variations.

\section{Conclusions}

We have experimentally tested both the gas permeability of a thin perfluorocarbon phase and its efficacy in stopping particulate contaminants. ${ }^{26} \mathrm{We}$ suggest that liquid barrier filters be constructed according to the guidelines given here as protective devices for conditions under which an atmosphere is charged with particulate contaminants so hazardous that a safe passage is normally possible only when carrying a supply of air. Neither respiratory filters of the fabric or matrix type nor positive pressure respirators can guarantee the complete elimination of particulate matter from the inspired air, as was shown recently in experiments with Thermoactinomycetes spores. ${ }^{10}$ Agents of an extremely high toxicity, contagiousness, or virulence contaminating an atmosphere can be assuredly eliminated by a liquid barrier filter that would guarantee adequate personal protection-for instance, against radioactive particulates released in a catastrophic nuclear event or against all types of micro-organisms disseminated in bacteriological warfare during the designed operational life of the filter unit.

We thank the Institut de recherche en santé et en sécurité du travail du Québec for financial support. 


\section{References}

' Clark LC, Gollan F. Survival of mammals breathing organic liquids equilibrated with oxygen at atmospheric pressure. Science 1966;152:1755-6.

${ }^{2}$ Seufert WD, Bessette F, Lachiver G, Merdy H. The liquid barrier filter-a new concept to eliminate particulate contaminants from gases. Health Physics 1982;42:209-16.

${ }^{3}$ Davies CN. Air filtration. London: Academic Press, 1973.

${ }^{4}$ Theodore L, Buonicore AJ. Industrial air pollution control equipment for particulates. Cleveland: CRC Press, 1976.

${ }^{5}$ Wesseler EP, Iltis R, Clark LC. The solubility of oxygen in highly fluorinated liquids. Journal of Fluorine Chemistry
1977;9:137-46.

- O'Brien RN, Langlais AJ, Seufert WD. Diffusion coefficients of respiratory gases in a perfluorocarbon liquid. Science 1982;217:153-5.

' Sargent JW, Seffl RJ. Properties of perfluorinated liquids. Federal Proceedings 1970;29:1699-1703.

${ }^{8}$ Clark JM, Schwabe PH. Air filtering respirators. In: Ballantyne B, Schwabe PH, eds. Respiratory protection. London: Chapman \& Hall, 1981

- Clark LC, Kaplan S, Becattini F. The physiology of synthetic blood. J Thorac Cardiovasc Surg 1970;60:757-73.

${ }^{10}$ Lacey J, Nabb S, Webster BT. Retention of actinomycete spores by respiratory filters. Ann Occup Hyg 1982;25:351-63. 